\title{
Transportation of liquid and sand in open trays
}

\author{
Irina Dezhina* and Vladimir Orlov \\ Moscow State University of Civil Engineering, Yaroslavskoe shosse 26, Moscow, 129337, Russia
}

\begin{abstract}
Herein there are theoretical dispositions on the conditions of the fluid flow and sand transportation within a wide range of rates. The results of some exploratory research on the sand transport in specially designed laboratory and production benches, including a platform with interchangeable modular polymer elements having an artificial rough surface, have been presented. The ranges of the experiment procedure have been established to create the turbulence zones, where the tray inner surfaces are of various texture in the form of obstacles of different height. The technique has been presented for carrying out experiments on largesize benches with modular pipe elements based on the use of photo and film equipment, as well as a set of devices for determining rates, filling and slopes. The results of hydraulic experiments on modeling the process of vortex formation in the flow in the trays with artificial roughness are presented. The ability of the flow to move sand dunes in the trays with an alternative texture of the inner surface has been studied, and the design data of the quantitative assessment of the transporting ability of the flow depending on various factors has been given.
\end{abstract}

\section{Introduction}

During transportation of a double-phase water flow containing a significant concentration of suspended solids, four flow regimes can be observed depending on the liquid flow rate $[1,2]$. At low fluid flow rates the sand deposits and forms a dense and displacementresistant sediment layer at the bottom of the pipe. With a bigger flow rate the sand begins to move in the form of a thin layer over the compacted sediment. At the further rate increase the compacted sediments, which have been accumulated at the bottom, begin to disintegrate into slowly flowing dune-type formations, which can increase both in area and volume in the form of vortex zones. When the rate grows up to the self-cleaning (critical) one, the sand particles almost completely pass into a suspended state in the fluid flow. The above mentioned four fluid flow modes were the result of the study of alluvial river channels [3]. As to the gravity drainage networks this was one of the basic elements for the study of transport processes in wastewater containing suspended solids. If we formulate briefly, the first parameter characterizing the nascent motion of sediment particles on the tray surface of pipelines is called the Shields number, the second is the gravity, the third is the filling and the fourth is the viscosity.

As evidenced by earlier experimental and theoretical studies of this subject, the threshold values determined theoretically in accordance with the bottom displacement rates, as a rule, are greater than these ones revealed from visual observations [4, 5]. This

\footnotetext{
* Corresponding author: dejina07@mail.ru
} 
discrepancy is largely due to the difference in the initial states of the sand crumpled in the tray part of the pipe [6].

It should be noted that the formation of a crumpled dense layer of sediment in the tray part of the pipeline can lead to certain risks, for example, an increase in friction losses, the probability of microbiological corrosion under the deposited layer, as well as the need more frequent cleaning of pipelines due to the failure of the relevant equipment, which increases the cost of operation of drainage networks [7, 8].

Improving the efficiency of removal of contaminants deposited on the tray part of the pipeline can be ensured by creating conditions (measures) that increase the flow turbulence, which contributes to the process of sediment transportation [9]. Thus, conducting field experiments to improve the transporting ability of drainage networks can be considered as an actual direction of scientific research and an extremely important aspect in the development of projects for the laying and reconstruction of pipelines, the use of modern systems for their diagnosis [10].

The wide possibilities of modern pipe production technologies and internal protective coatings of composite materials with almost any surface structure contribute to the solution of the assigned tasks, especially in case of application of trenchless methods of repair of engineering systems $[11,12]$ and the use of new repair materials [13].

\section{Materials and methods. Conducting preliminary (exploratory) research on the sand transportation in trays with different texture of their inner surface on small benches}

Open chutes (pipe trays), $130 \mathrm{~mm}$ diameter, with an artificially created textured (corrugated) surface were used as the object of the field research. The trays made an integral part of the experimental small-sized hydraulic unit developed by the authors of the article [14]. The bench enabled creation of the conditions, which provided a double-phase flow turbulence and the intensity of sand inclusions removal with the diameter of fractions 2.5, 1.5 and $0.3 \mathrm{~mm}$ in wide ranges of the flow rates at the minimum slope of the tray 0.07 and the water layer heights within 1-4 mm, i.e. proportionate or slightly greater than the diameters of the sand fractions.

The corrugated surfaces were made as several different types of grouped polymer obstacles of different shapes (round and in the form of parallelepipeds with a semicircular, rectangular and pointed section, pyramidal and in the form of corners), located on the bottom of the tray on both sides of its axis. The diameter of the round obstacles ranged from 2 to $7 \mathrm{~mm}$ with a height of $2 \mathrm{~mm}$, and the dimensions of the parallelepipeds made $20 \times 2 \mathrm{~mm}$ and a height of $2 \mathrm{~mm}$. The experiments were accompanied by filming and photographing (by Sony $\alpha 550$ digital SLR camera, DT 1.8 / 50 SAM lens) with creation of the black and white (shade) effect by a special lamp. It enabled the tracing and recording the picture of the flow turbulence occurrence as well as the sand particle removal.

In table 1 , as one of the characteristic examples, the results of experiments on visual observation of sand removal dynamics with simultaneous measurement of water flow rates along the tray using one of the types of corrugations in the form of parallelepipeds are presented. 
Table 1. Hydraulic and geometrical parameters of the first simulation case.

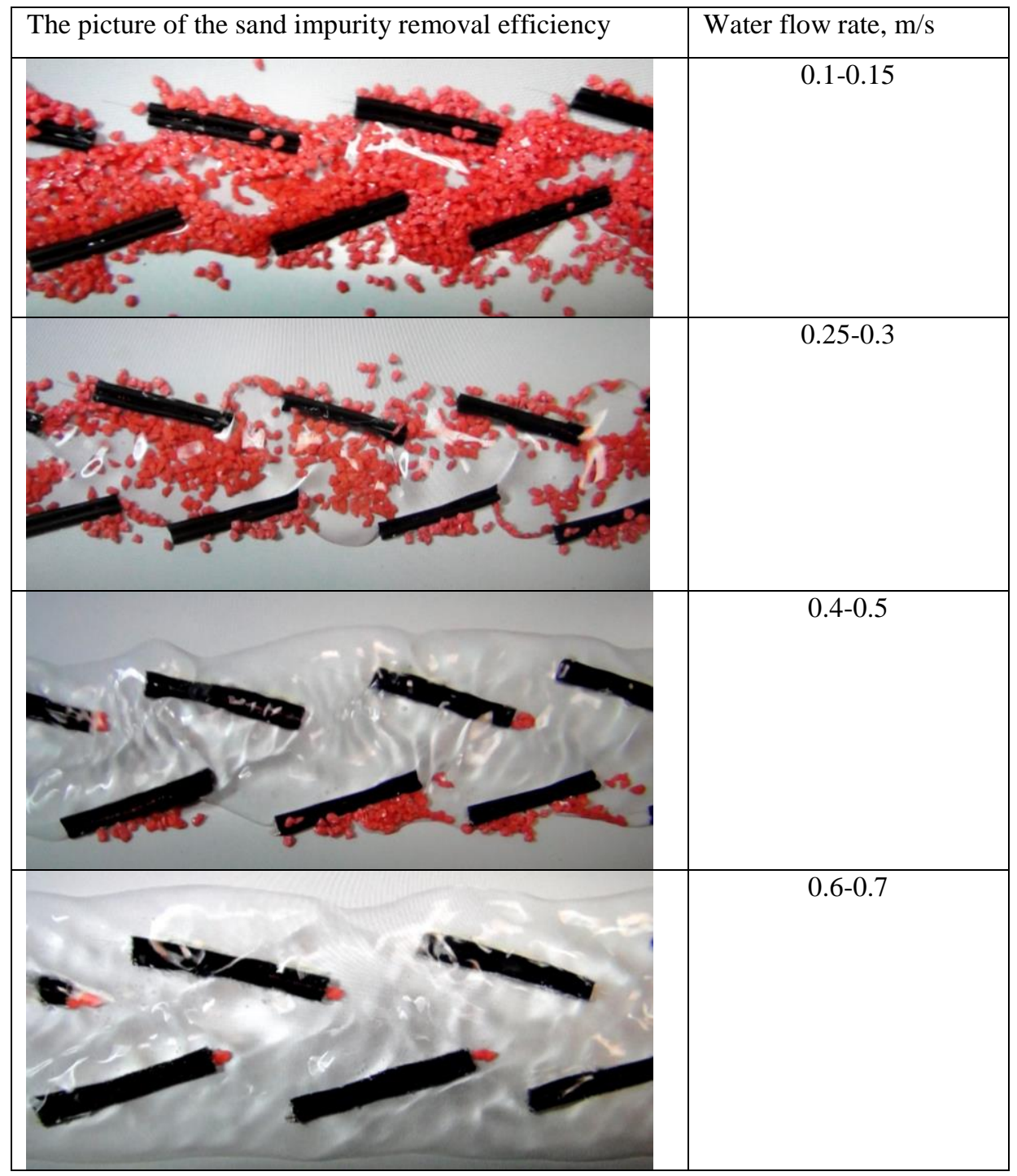

The analysis of the got results made it possible to conclude, that when the water flow rate in the tray is close to the critical one (about $0.7 \mathrm{~m} / \mathrm{s}$ ) there is a complete removal of sand particles. The use of other types of artificial corrugating obstacles enabled the similar effect. The results of exploratory field experiments led to expansion of the turbulization process to a full-scale modeling of the of single-phase and double-phase gravity flow on a large bench with big fillings, a wide range of rates and the use of various configurations of obstacles that form an artificial roughness. 


\section{Results and discussions. Experimental studies of the microturbulence occurrence on a large bench and development of approaches to the assessment of the flow transporting ability}

The production stand in the form of a pipeline with a diameter of $150 \mathrm{~mm}$ and a total length of $10 \mathrm{~m}$ included a manhole $(2.5 \mathrm{~m}$ long) in the form of an open chute, in which separate tray modules (of $130 \mathrm{~mm}$ diameter) with applied artificial corrugations were alternately placed. As accessories, the same set of equipment was used as during the work at the laboratory bench.

During the experiments, the range of the filling and flow rates has been changed for different types of textured surfaces, in particular, of the following shapes: frontal rectangular; semicircular, rectangular pointed (with different angles) and curvilinear pointed (with different angles).

The experiments were preceded by the search for operating ranges, i.e. a set of hydraulic flow parameters within a certain range of the water flow rates and fillings in the pipeline with artificially created roughness of the pipeline inner wall. The solution of the problem was based on the semi-empirical theory of turbulence, on the basis of which the optimal zone for field experiments on an open tray was determined through a universal index, expressed as a criterion of the $R_{e}{ }^{*}$ turbulence zone or the reduced Reynolds number $[15,16]$.

The experiments have shown that in the range of water flow rates $0.2-0.8 \mathrm{~m} / \mathrm{s}$ through a pipe with a diameter of $130 \mathrm{~mm}$ and a height of roughness projections in the range of 1-4 $\mathrm{mm}$ with a space of $10 \mathrm{~mm}$, as well as the kinematic viscosity of the liquid $v=1.110710^{-6}$ $\mathrm{m}^{2} / \mathrm{s}\left(\right.$ at $16^{\circ} \mathrm{C}$ ), the turbulent motion is observed at the Reynolds number $R_{e}$ more than 800 $[17,18]$. As a result of the made calculations, the optimal area of the field investigations has been determined. It is presented in the form of a green zone in figure 1.

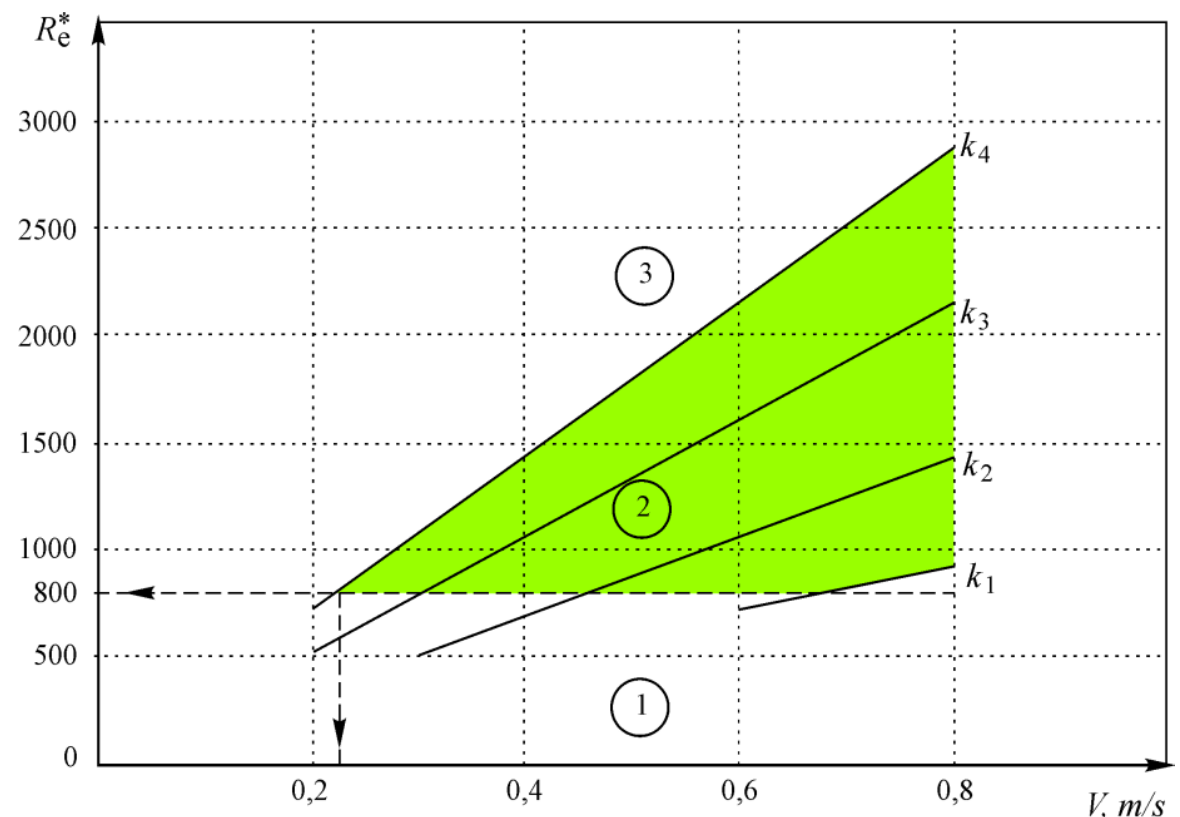

Fig. 1. Illustration of the experimental zone in the range of possible rates at the obstacle heights: $k_{1}=1 \mathrm{~mm}, k_{2}=2 \mathrm{~mm}, k_{3}=3 \mathrm{~mm}$ and $k_{4}=4 \mathrm{~mm}$

1-laminar flow zone for any obstacles within the considered range, 2-experimental zone; 3 turbulent flow zone for obstacles higher than $4 \mathrm{~mm}$ 
Subsequently, the experiments were carried out by reproducing two hydraulic modes, i.e. single-phase (water without sand inclusions) and double-phase (liquid with sand inclusions) flows. The goal was to investigate the laminating (dissipative) effect of fine impurities on the vortex formation during the transition to the turbulent flow [19, 20].

The essence of the method of the double-phase flow investigation consisted in backfilling of the standard weight amount of sand of a certain fractional composition to the bottom of the pipe module on a certain area (with the appropriate length and width). Thus, an artificial sand dune has been formed. The study was subject to the process of movement of individual particles of sand along the bottom of the tray, which separate from the dunes at when increasing the rates and the filling. In parallel, the transporting ability of the flow has been determined at different textures of the tray inner surface and the sand particle size while simultaneously measuring the flow hydraulic characteristics, in particular the flow rate and the filling.

It should be noted, that during the experiments with single-phase and double-phase flows, the nature of vortex formation on the flow surface in the pipe module has to be studied, as well as the rate (by float method) and the filling (by measuring ruler) have been measured. In both cases, a characteristic feature was the use of a light source with a special luminaire of two parallel lamps in the form of tubes, which were contributing to the creation of reflection and deformation picture on the water mirror of the chiaroscuro path (vortex cord simulator), fixed by a coaxial photo camera.

The Table 2 presents one of the results of a series of single-phase flow experiments for the following conditions: the type of obstacle - consistently arranged wedges or springboards (bars with 1-4 mm variable height), forming a system (50 mm long), including the first and second stages with pointed ends of wedges to the flow. This type of location obstacles was called «the form of a Christmas tree».

The aim of the experiments was to study the dynamics of microturbulence manifestation by means of the shade effect in a wide range of rates and fillings.

Table 2. Visualization of the flow and its characteristics.

\begin{tabular}{|c|c|c|c|c|c|}
\hline \multirow{2}{*}{$\begin{array}{c}\text { Experi- } \\
\text { ment } \\
\text { number }\end{array}$} & \multicolumn{3}{|c|}{ Values of time, flow rate, water layer height and filling } \\
\cline { 2 - 6 } & $\begin{array}{c}T_{a v}, \mathrm{~s} \\
\mathrm{per} \\
1 \mathrm{~m})\end{array}$ & $\begin{array}{c}\text { Rate } \\
V_{a v}, \\
\mathrm{~m} / \mathrm{s}\end{array}$ & $\begin{array}{c}\text { Height } \\
h_{a v}, \mathrm{~mm}\end{array}$ & $\begin{array}{c}\text { Filling } \\
h / d_{a v}\end{array}$ & Picture showing the state in the tray \\
\hline 1 & 6.5 & 0.154 & 7 & 0.054 & \\
\hline 2 & 6.3 & 0.158 & 10 & 0.077 & \\
\hline
\end{tabular}




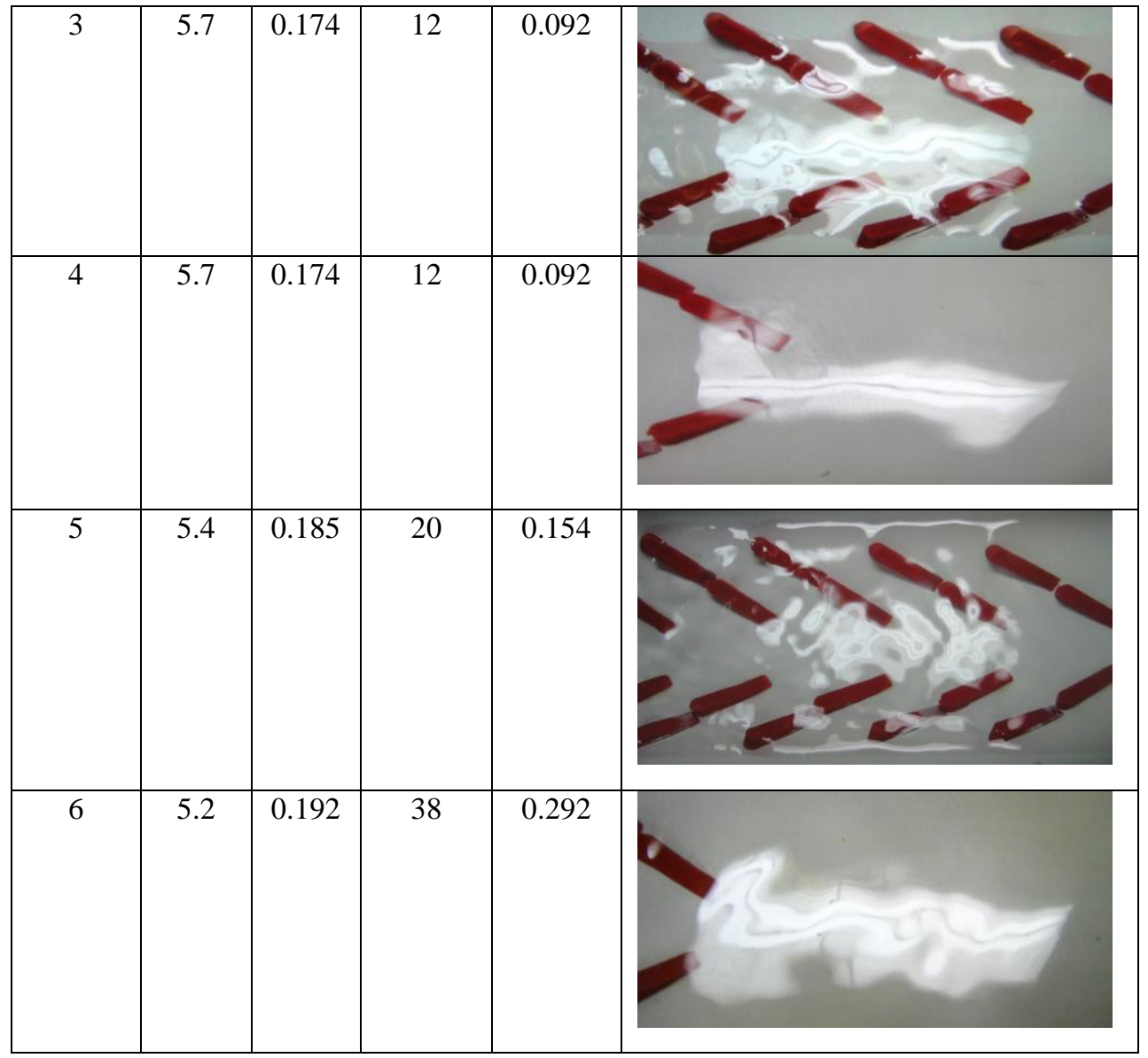

Interpretation of the got results of 6 experiments is presented below.

Experience 1. The water edge is at the level of the upper edge of the first step; the shadow line (light cord) is smooth at a speed of $0.154 \mathrm{~m} / \mathrm{s}$ in the middle of the obstacle zone.

Experience 2. The water edge is close to the upper edge of the second step; the shadow line began to undergo slight deformation (bending) in the middle of the obstacle zone with an increase in speed to $0.158 \mathrm{~m} / \mathrm{s}$.

Experience 3. The water edge is almost at the level of the upper edge of the second step; the shadow line is wavelike deformed in the middle of the zone with obstacles at a rate of $0.174 \mathrm{~m} / \mathrm{s}$;

Experience 4. Identical to experience 3 by the values of rates and fillings; however, the difference is that before the obstacles the shadow line is smooth (weak turbulization), and in the middle it is stronger. Thus, it can be stated that the presence of obstacles causes an increased microturbulence.

Experience 5. The water edge is above the upper edge of the second step; complete violation of the shadow line; the movement is classified as coherent, starting at a speed of $0.185 \mathrm{~m} / \mathrm{s}$;

Experience 6. The water edge is above the upper edge of the second step; the shadow line is deformed wave-like only at a speed of more than $0.192 \mathrm{~m} / \mathrm{s}$; comparison with the results obtained in the fifth experiment indicates that the transition from vortex to coherent motion does not still occur before the obstacle despite the relatively high rate. Thus, it can 
be stated once again that the presence of obstacles at approximately equal water flow rates leads to a micro-turbulence in comparison with the cases of the obstacle absence.

Similar experiments were carried out at the front resistance as well, when the obstacles were placed by not pointed but the wide side towards the flow. The results showed the nature of the turbulence occurrence close to the above described one.

Subsequent experiments on the study of the double-phase flow were carried out with another types of obstacles and different fractional composition of the sand, in order to determine the flow transporting ability. At the same time, the mass of the sand ridge, which has been evenly distributed over an area of $76.5 \mathrm{~cm}^{2}(17 \times 4.5 \mathrm{~cm})$ between the obstacles, was assumed to be standard and equal to $28 \mathrm{~g}$, and the diameter of the sand fraction varied in the range of $0.3-3.0 \mathrm{~mm}$.

The Table 3 presents the results of one of the experimental series with obstacles in the form of wedges (with a high frontal end of the semicircular profile towards the flow direction. The diameter of the sand fractions was $2.5-3.0 \mathrm{~mm}$.

Table 3. Visualization of the flow and its characteristics.

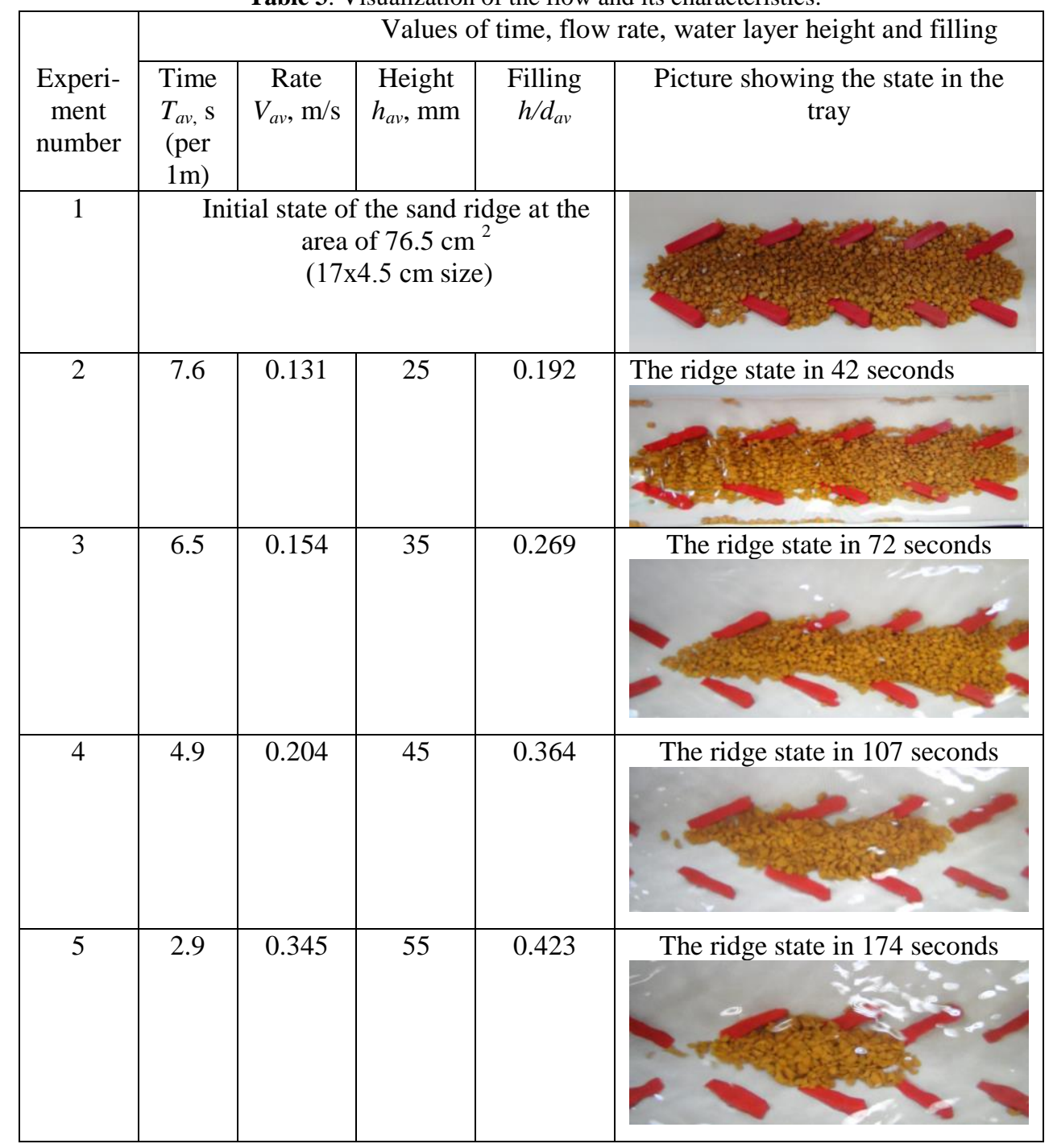




\begin{tabular}{|c|c|c|c|c|c|}
\hline 6 & 2.9 & 0.345 & 58 & 0.446 & The ridge state in 237 seconds \\
& & & & & \\
\hline 7 & 2.9 & 0.345 & 58 & 0.446 & The ridge state in 257 seconds \\
\hline 8 & 2.9 & 0.345 & 58 & 0.446 & The ridge state in 274 seconds \\
\hline & & & & & \\
\hline
\end{tabular}

In the initial stages (experiments 2-3) the rate was $0.131-0.154 \mathrm{~m} / \mathrm{s}$, and the filling 0.192-0.269. In subsequent experiments (4-8) the rate and the filling were constant, respectively $0.345 \mathrm{~m} / \mathrm{s}$ and 0.446 .

The complete removal of sand from the area of the sand dune occurred in 4 min $34 \mathrm{~s}$ or 274 s. Hence, the relative transporting ability made: $\mathrm{cm}^{2}$

A) per unit of the dune area: $28 / 274 / 76.5=0.0013358 \mathrm{~g} / \mathrm{s}$ per $\mathrm{cm}^{2}$ or $1.336 \mathrm{mg} / \mathrm{s} \mathrm{per}$

B) per unit of the dune length: 28/274/17 $=0.00601 \mathrm{~g} / \mathrm{s} \mathrm{per} \mathrm{cm}$ or $6.01 \mathrm{mg} / \mathrm{s} \mathrm{per} \mathrm{cm}$.

A comparative analysis of the transport capacity in the presence of obstacles (wedges) with a pointed end towards the flow and a semicircular frontal edge states the proximity of the got results:

-the range of rates for the two cases makes $0.345-0.5 \mathrm{~m} / \mathrm{s}$ and the fillings of 0.377 0.446 ;

-the range of transporting abilities: respectively to the dune areas $1.336-1.482 \mathrm{mg} / \mathrm{s} \mathrm{per}$ $\mathrm{cm}^{2}$ and the dune lengths of $6.01-6.67 \mathrm{mg} / \mathrm{s}$.

Thus, the location of obstacles (wedges) with a pointed end to the flow or a semicircular frontal wedge have not been significant for the transportation of sand ridges. Other types of obstacles (pyramids, angles) did not increase the transportation effect.

\section{Conclusion}

1. The technique of full-scale experiments has been developed for the study of the hydraulic parameters of single-phase and double-phase flows in open trays with a textured surface (in the form of wedges with pointed and frontal resistance, pyramids, angles, etc.). The range of fillings was within 0.05-0.6 and the fluid flow rates within 0.1-0.6 m/s. As the object of transportation provision has been made of sand of different particle size (0.1-3.0 $\mathrm{mm}$ ), washed from an artificially created dune.

2. Using the shade effect of a luminaire with two parallel lamps, the regularities of the fluid flow movement (laminar, turbulent in the form of vortex and coherent character) have been revealed. On the basis of visual observations and the calculated parameters of singlephase flows, it has been established that there are no fundamental differences in the location 
of various types of obstacles in the tray and their numerous combinations to create microturbulence. A coherent flow occurs at the rates beginning from $0.3 \mathrm{~m} / \mathrm{s}$ and fillings greater than 0.3, which was used as the basis for experiments on the double-phase flow.

3. The estimation of the tray transporting ability at a two-phase flow for the removal of bulk (non-cohesive) sand impurities has been made. The specificity of the sand removal by rolling and transition to the suspended state is noted. As a result of experiments in trays with different corrugated surfaces and a wide range of the sand fraction diameters, the most effective forms of obstacles (wedges with a pointed end to the flow) and options for their location (for example, in the form of a «Christmas tree») are established, allowing to achieve an effective transporting ability, estimated as the removal of sand mass per unit of the dune area $\left(\mathrm{mg} / \mathrm{s}\right.$ per $\left.\mathrm{cm}^{2}\right)$ and per unit of the dune length $(\mathrm{mg} / \mathrm{s} \mathrm{per} \mathrm{cm})$.

4. It has been experimentally proved that within the specified rate values $(0.2-0.8 \mathrm{~m} / \mathrm{s})$ the roughness projections $(1-4 \mathrm{~mm})$ do not have a significant effect on the transporting ability of the flow. Moreover, the presence of corrugated surfaces reduces the frequency of regular cleaning of pipelines, counteracting the formation of stagnant sand ridges.

5. As a result of experimental studies, it was found that in various situations associated with the type and location of obstacles and the size of the sand, the flow transporting ability to remove fractions with a diameter of $0.1-0.3 \mathrm{~mm}$ compared to the diameter of the fractions of 2.5-3.0 mm is significantly different. A greater effect is observed in the removal of fine sand fractions. At the same time, the difference in the transporting ability per unit of area is about $53.1 \%$, and per unit of the dune length is $46.6 \%$. Obstacles in the form of pyramids and corners are ineffective, because they do not provide a complete removal of sand ridges from the surface of the tray.

\section{References}

1. Arolla S.K., Desjardins O. Transport modeling of sedimenting particles in a turbulent pipe flow using Euler-Lagrange large eddy simulation. International Journal of Multiphase Flow. 2015. p.734-750p.

2. Danielson T.J. Sand transport modeling in multiphase pipelines. International Offshore Technology Conference (2007)

3. Church M. Bed material transport and the morphology of alluvial river channels Annual Review of Earth and Planetary Sciences (2006), 34 (1),p. 325-354.

4. Kuliczkowski A., Rury kanalizacyjne. Wydawnictwo Politechniki Swietokrzyskiej. (2004). 507 p.

5. Idelchik I.E. Flow Resistance: A Design Guide for Engineers. Routledge. 2017. 416 p. (rus)

6. Dancey C.L., Diplas P., Papanicolaou A, Bala M. Probability of individual grain movement and threshold condition. Journal of Engineering, 128(2), (2002), p. 10691075

7. Grossmann S., Lohse D. Curvature effects on the velocity profile in turbulent pipe flow Eur. Phys. J. E. No. 40 (2017)

8. Ebtehaj I., Bonakdari H. Comparison of genetic algorithm and imperialist competitive algorithms in predicting bed load transport in clean pipe.Water Sci Technol, (2014), N. 70(10), p. 695-701

9. Santiago A., Durango M. Most advanced technology for pipeline inspection in the world: see, measure and navigate in $3 D$ through pipes and manholes. International Conference and Exhibition NO-DIG 2012, Sao Paulo (Brasil)

10. Ahern E. Non-invasive rehabilitation technologies. 30 NO-DIG International Conference and Exhibition, Sydney (Australia) (2013) Paper 2-20, pp. 1-9.

11. Rabmer-Koller U. No-dig technologies - innovative solution for efficient and fast pipe 
rehabilitation.29 NO-DIG International Conference and Exhibition, NO-DIG Berlin (2011) Paper 2C-1, p. 1-10

12. Janssen A. Importance of lateral structural repair of lateral lines simultaneously with main line CIPP rehabilitation. 30 NO-DIG International Conference and Exhibition, (2012) Sao Paulo (Brasil), PAP 012287, p. 1-9

13.. Greaves G.N., Greer A.L., Lakes R.S., Rouxel T. / Poisson's ratio and modern materials. J. Nature Materials. (2011). vol. 10, p. 823-3

14. Orlov V.A., Dezhina I.S., Pelipenko A.A., Orlov E.V. Test bench for the study of the flow of liquid by optical means in open trays with different relief of their inner surface. Patent of the Russian Federation for utility model (2018) No. 176330

15. Orlov V.A., Dezhina I.S., Korolyov A.A. Determination of boundary values of the field of turbulence for hydraulic experiments on pipelines with the text of the inner surface.Vestnik MGSU. 2018, Volume 13. No. 5 (116). p. $426-434$ (rus)

16. Altschul, A.D., Calitsun V.I., Mayranovsky F.G. Primery rasschetov po gidravlike [Examples of calculations for hydraulics]. Moscow: Alliance. (2013) 255 p. (rus)

17. Altschul, A.D., Calitsun V.I., Mayranovsky F.G. Primery rasschetov po gidravlike [Examples of calculations for hydraulics]. Moscow: Alliance. (2013) 255 p. (rus)

18. Bryanskya Yu. V. Refinement of the cinematic characteristics of the turbulent flow Civil engineering Journal. (2013) 6 (41). p. 31-38

19. Dulin V.M., Kozorezov Yu.S., Markovich D.M. Evaluation of turbulent kinetic energy dissipation rate in a free jet flow from PIV measurements. Bulletin of NSU, Series: Physics (2012) Volume 7. p. 53-69 (rus)

20. Girgidov A.D. Izmenenie dissipacii energii pri perehode ot laminarnogo rezima $\mathrm{k}$ turbulentnomy [Changing of energy dissipation during flow transformation, from laminar to turbulent] Inzhenerno-stroitelniy zhurnal. (2011) No. 5 (23). p. $49-52$ (rus) 